\title{
JOINT FOURIER AND NON-FOURIER SPECTRAL / PSEUDO-SPECTRAL APPROACH TO THE LUNG BIOACOUSTICS AND BIOMEDICAL SIGNAL FINGERPRINTING AS A WAY TO INCREASE THE QUALITY OF THE LUNG DIAGNOSTICS USING SUPERCOMPLEX HYBRIDIZATION OF DIFFERENT DSP METHODS
}

\author{
Eugeny D. Adamovič and Oleg V. Gradov \\ Institute for Energy Problems of Chemical Physics, Moscow, Russia \\ O.V. Gradov \\ 38/2 Leninsky pr., Moscow, Russia, 119334 \\ E-mail: neurobiophys@gmail.com
}

\begin{abstract}
A novel approach to the multifactor / multi-variance analysis of lung sounds has been developed and implemented into the experimental biomedical practice. This approach is based on the information decision, extracted from different spectral or pseudo-spectral analysis results, including non-Fourier spectra, Prony energy spectra, moving average spectra, autoregressive frequency spectra, minimum variance frequency spectra, eigenanalysis frequency estimation, Lomb periodograms, etc. The above approach reduces the degree of uncertainty of the analysis due to consideration of the additional diagnostic information derived from the standard raw data (files) of the pulmonary signal registration. The application of the novel complex mathematical methods of data processing allows for the first time to extract a number of important and complex descriptors which were previously ignored due to their complexity, but now can be easily interpreted using the novel data mining approaches.
\end{abstract}

Keywords: lung bioacoustics, multifactor or multi-variance analysis, pseudo-spectral analysis, complex descriptors, biomedical signal registration, digital signal processing 


\section{Introduction}

Since 1980-th digital Fast Fourier Transform (FFT) methods have been frequently used in lung bioacoustics and acoustic breath diagnostics (Rhodes, 1979; Charbonneau, 1983; Gavriely, 1985). Simple computer-based measurements and systems for analysis of the pulmonary auscultation sounds hitherto apply computing of the FFT patterns for displaying of the power spectrum and spectrogram (Polat, 2004). Sometimes FFT is complemented by the more complex methods of analysis, such as parameter estimation methods in particular autoregressive (AR) modeling (Gavriely, 1985), classification of the lung sounds in patients with asthma, emphysema, fibrosing alveolitis and healthy lungs by using self-organizing maps (Malmberg, 1996) and principal component analysis (Mussel, 1990).

However, single Fourier transform and its analogs, as well as its combination with any other single method is not sufficient for the diagnostic purposes. The review (Malone, 1993) compares five methods that utilize electronic / computer acoustic processing techniques (cited from (Malone, 1993)): "The first method uses traditional spectrographic techniques to produce time / frequency / intensity three-dimensional representation of the waveform. The second method is computer-based and uses the fast Fourier transformation (FFT) to show the frequency composition of the waveform. The third uses linear prediction coefficients (LPCs) to produce a power spectrum and inverse filtering to estimate the cross-sectional area of the human upper airway. The fourth technique employs a proprietary digital filterbank ..., which may be used as a control by subsequent researchers. In the fifth method, a physiologically based digital filterbank, designed to closely model the human ear response, is proposed. It is envisaged that this approach will offer the flexibility of all the previous techniques and also closely model the analysis procedure carried out using subjective auscultation". Finally the authors arrive to a conclusion that "... none of the above techniques are sufficiently robust to provide unambiguous diagnosis ... and that a reappraisal is required in terms of feature extraction so that relevant features can be identified" (Malone, 1993). In this regard (despite the fact that such a conclusion has been made not for all the breath bioacoustics phenomena containing the phases of regular and chaotic dynamics), it is necessary to develop a set of descriptors allowing to perform complex diagnostics independently from the subjective human factors. This research trend involves multivariate analysis and combination of many spectral processing methods ("bioacoustic spectroscopy" / "bioacoustic fingerprinting"), as well as the development of the heuristic models of pulmonary respiration, which meet the requirements of the Fourier analysis and possess a full set of variables which is not used in the conventional simplified simulation methods with the diagnostics of their similarity to the biological prototype according to the Fourier spectra (FFT) (Jones, 2000). Moreover, this provides new ways for the development of the lung sound characterization after adaptive filtering (Ploysongsang, 1989).

\section{Our Open Source investigations and proposals}

We suggest an Open Source product (for example - for Linux), where the decision-making and the diagnostics of the lung pathologies are based not only on the conventional measuring methods of the lung sound diagnostics, but also on a set of values derived from the following methods with graphical visualization:

a) Auto-regressive frequency spectra and visualization of the: root summary (in coordinates: $\mathrm{X}=\mathrm{Re}, \mathrm{Y}=\mathrm{Im}$ or $\mathrm{X}=$ sorted index, $\mathrm{Y}=$ magnitude); order selection criteria (in coordinates: $\mathrm{X}=$ order, $\mathrm{Y}=$ normalized criterion); autoregressive residuals (stabilized normal probability plot; histogram: $\mathrm{X}=$ standard deviation, $\mathrm{Y}=$ counts; "percent residuals" as a $\%$ of the $\mathrm{Y}$ data value; standardized residuals - as a fraction of the fit's standard error; basic residuals - the simple difference between the $\mathrm{Y}$ data value and the $\mathrm{Y}$ prediction from the fit).

b) Prony energy spectra (computed using either damped or undamped algorithms based on different signal subspaces with the variety of orders) and visualization of the: autoregressive residuals (see above), signal threshold selection based on the singular value decomposition (in 
coordinates: $\mathrm{X}=$ index; $\mathrm{Y}=$ singular value) and the root summary (in coordinates: $\mathrm{X}=\mathrm{Re}, \mathrm{Y}=\mathrm{Im}$ and $\mathrm{X}=$ sorted index, $\mathrm{Y}=$ magnitude).

c) Lomb / Lomb-Scargle periodogram (in coordinates: $\mathrm{X}=$ time, $\mathrm{Y}=$ normalized power Vs. Y = magnitude Vs. Y = amplitude) with non-linear optimization and non-linear numerical fitting (with the built-in variables, such as rate, phase, frequency and amplitude). Raster analysis of the regularity and periodicity.

d) Eigendecomposition (in coordinates: $\mathrm{Y}=$ eigenvalue, $\mathrm{X}=\mathrm{index}$ ) and visualization of the eigenvector graphics $(\mathrm{Y}=$ eigenvector, $\mathrm{X}=$ index $)$, principal components $(\mathrm{Y}=$ principal component; $\mathrm{X}=$ index $)$, computational eigendecomposition data reconstruction $(\mathrm{Y}=$ signal, $\mathrm{X}=$ time), FFT and autoregressive frequency spectra reconstructions using eigendecomposition methods.

e) Autocorrelation (in coordinates: $\mathrm{Y}=$ autocorrelation, $\mathrm{X}=\mathrm{lag}$ ), including exotic graphical representations, such as autocorrelation graphs with logarithmic $\mathrm{X}$ or / and $\mathrm{Y}$ axes and also logarithmic $1 / \mathrm{N}$ representation. Crosscorrelograms with the shift-predictions and reverse correlation analysis diagrams.

f) Moving average frequency spectra (in time integrated square analysis PSD mode power spectrum density graphical representation) and ARMA (autoregressive moving average) spectra.

g) Time domain reconstruction (in coordinates: signal amplitude $=\mathrm{Y}$; time $=\mathrm{X}$ ) and direct Fourier domain filtering with the Fourier spectrum calculation, fitting and visualization (in coordinates: $\mathrm{X}=$ frequency; $\mathrm{Y}=$ amplitude Vs. phase Vs. magnitude Vs. variance Vs. $\mathrm{dB}$ or normalized $\mathrm{dB}$ [i.e. power spectrum density or the RMS-like parameter]) compared with the spectra of the data tapering windows.

h) Parametric spectra and parametric reconstructions of the lung sounds and breath registration signals based on different signal subspaces in the frames of either undamped or damped sinusoidal models. These approaches may be implemented not only to the FFT spectral analysis, but also to autoregressive spectra, Prony energy spectra and Lomb / Lomb-Scargle periodograms.

i) FFT spectral analysis of any converted and processed data formats, including Doppler shifted registrations, Savitzky-Golay smoothed / filtered data (including $1^{\text {st }}, 2^{\text {nd }}, 3^{\text {rd }}$ and $4^{\text {th }}$ derivatives), spline interpolated / estimated data (including $1^{\text {st }}$ and $2^{\text {nd }}$ derivatives) and also noised, distorted and convolved registration data (including time-code-consisted files).

j) Linear prediction of the signal structures using different signal subspaces, any specific orders (min, max, inc) and any stabilization regimes (without stabilization, reflection-based output stabilization, circle feedback stabilization, etc.). Fractal analysis, including Hurst exponent and some variants of the Hurst exponent graphical representation and also the phase space, correlation integral, correlation dimension \& correlation entropy graphics.

k) Interstitial phenomena analysis in terms of the nonlinear theory and radio-electronic digital signal processing: total harmonic distortion, intermodulation distortion, equivalent noise levels, signal-to-noise ratios, delay / decay measurements, increments / decrements of the raw signal oscillations.

1) Poincare maps of intervals, stereogoniometric graphs of the phase and cross-channel correlations in polar and elliptical coordinates, vector representation of the phase analysis and the phase analysis using the Hilbert transform.

m) Integral estimation of the lung volume by the signal analysis and the fluctuation rate or / and noise instability analysis by means of the cyclic code-like techniques.

\section{Conclusions}

We have previously performed the above data processing and data mining with the existing software, which failed to provide the direct diagnostic measurements, while the precious positioning of the measurement results within the frequency range allowed us to perform the data comparison in the manual mode, which was sufficient for the purposes of the ornithological, ethological and 
biogeographical bioacoustics (Gradov, 2013; Gradov, 2015)]. The development of the specialized software capable of performing the above listed functions automatically in a single GUI with the option providing the multivariate / multifactor data clustering into the diagnostic groups) can provide new insights to the lung bioacoustics.

\section{References}

1. Charbonneau G, Racineux JL, Sudraud M, Tuchais E. An accurate recording system and its use in breath sounds spectral analysis. J Appl Physiol Respir Environ Exerc Physiol 1983, 55(4):11201127.

2. Gavriely N, Herzberg M. Parametric representation of normal breath sounds. J Appl Physiol 1985, 73(5):1776-1784.

3. Gradov O. Bioacoustical fingerprinting as a multifactor method for automatic identification of avifauna. In Proceedings of the IV International Buturlin Conference: 19-22 September 2012; Ulianovsk. Edited by Yu.Volodina, O. Borodina, T. Gromova, D. Korepova; 2012:65-74 [Russian]. 4. Gradov OV, et al. Bioacoustical correlometers for ornithologists. Multi-Preprint, DLM, INEPCP, 2015.

5. Jones AY, Jones RD, Kwong K, Burns Y. The effect on sound generation of varying both gas flow rate and the viscosity of sputum-like gel in a simple tubular model. Lung 2000, 178(1):31-40.

6. Malmberg LP, Kallio K, Haltsonen S, Katila T, Sovijärvi AR. Classification of lung sounds in patients with asthma, emphysema, fibrosing alveolitis and healthy lungs by using self-organizing maps. Clin Physiol 1996, 16(2):115-129.

7. Malone M, Black ND, Lydon M, Cinnamond M. Acoustic analysis of infantile stridor: a review. Med Biol Eng Comput 1993, 31(2):85-96.

8. Mussell MJ, Nakazono Y, Miyamoto Y, Okabe S, Takishima T. Distinguishing normal and abnormal tracheal breathing sounds by principal component analysis. Jpn J Physiol 1990, 40(5):713-721.

9. Ploysongsang Y, Iyer VK, Ramamoorthy PA. Characteristics of normal lung sounds after adaptive filtering. Am Rev Respir Dis 1989, 139(4):951-956.

10. Polat H, Güler I. A simple computer-based measurement and analysis system of pulmonary auscultation sounds. J Med Syst 2004, 28(6):665-672.

11. Rhodes DC. Bioacoustics fast Fourier transform processor. B.S.: University of Illinois; 1979. 